Western University

Scholarship@Western

Obstetrics \& Gynaecology Publications

Obstetrics \& Gynaecology Department

$3-1-2007$

\title{
Oocyte cytoplasmic maturation: a key mediator of oocyte and embryo developmental competence.
}

AJ Watson

Follow this and additional works at: https://ir.lib.uwo.ca/obsgynpub

Part of the Obstetrics and Gynecology Commons

Citation of this paper:

Watson, A J, "Oocyte cytoplasmic maturation: a key mediator of oocyte and embryo developmental competence." (2007). Obstetrics \& Gynaecology Publications. 35.

https://ir.lib.uwo.ca/obsgynpub/35 


\title{
Oocyte cytoplasmic maturation: A key mediator of oocyte and embryo developmental competence ${ }^{1}$
}

\author{
A. J. Watson ${ }^{2}$ \\ Departments of Obstetrics and Gynaecology and Physiology and Pharmacology, \\ The University of Western Ontario, Children's Health Research Institute, London, Ontario, Canada N6A 4G5
}

\begin{abstract}
Efforts have intensified to successfully mature and inseminate oocytes in vitro and then culture ensuing embryos to transferable stages from a large number of mammalian species. Success varies, but generally even for the most successful species it is only possible to obtain a maximum of a 40 to $50 \%$ development of zygotes to the blastocyst stage. Reduced oocyte developmental competence is suggested as a primary reason for the reduced potential of in vitro-produced embryos. The vast majority of in vitro-matured oocytes are meiotically competent; however, many do not attain an optimal oocyte diameter before insemination. Variations in oocyte in vitro maturation media can influence embryo development, blastocyst cell number, and apoptosis. In addition, studies have indicated that cytoplasmic donation from so-called competent to in-
\end{abstract}

competent oocytes can improve developmental outcomes. Oocyte cytoplasmic maturation includes those events that instill upon the oocyte a capacity to complete nuclear maturation, insemination, early embryogenesis and thus provide a foundation for implantation, initiation of pregnancy, and normal fetal development. Although we can define oocyte cytoplasmic maturation, we are only now beginning to understand the molecular steps that underlie this process. In general terms, oocyte cytoplasmic maturation involves the accumulation of mRNA, proteins, substrates, and nutrients that are required to achieve the oocyte developmental competence that fosters embryonic developmental competence. Collectively we are beginning to specify oocyte cytoplasmic maturation, and eventually a coherent understanding of this critical event in oocyte biology will emerge.

Key words: ovary, oogenesis, in vitro maturation, cumulus cell, preimplantation development, embryo transfer

(C2007 American Society of Animal Science. All rights reserved.

J. Anim. Sci. 2007. 85(E. Suppl.):E1-E3

doi:10.2527/jas.2006-432

\section{OVERVIEW}

The characterization of fully defined, optimized culture conditions for oocyte maturation and preimplantation development in vitro is an obvious priority, especially when there is increasing evidence demonstrating the negative consequences of oocyte and embryo culture on gene expression patterns and, more dramatically, on embryo and fetal health in animal models (BilodeauGoeseels and Panich, 2002; Fair et al., 2004; Krisher, 2004). These points are of particular importance as the human assisted reproduction technology field prepares to adopt in vitro oocyte maturation and zygote culture

\footnotetext{
${ }^{1}$ Presented at the ADSA-ASAS Joint Annual Meeting, Triennial Reproduction Symposium: The Follicle and the Oocyte, Minneapolis, MN, July 2006.

${ }^{2}$ Corresponding author: awatson@uwo.ca

Received July 4, 2006.

Accepted August 8, 2006.
}

to the blastocyst stage before embryo transfer as routine technologies.

In recent years, efforts have intensified to successfully mature and inseminate oocytes in vitro and then culture the ensuing embryos to transferable stages from a large number of mammalian species (Gilchrist et al., 2004; Krisher, 2004; Sirard et al., 2006). For the most successful species, it is only possible to obtain development of a maximum of 40 to $50 \%$ of the zygotes to the blastocyst stage. Reduced oocyte developmental competence is suggested as a primary reason for the reduced potential of in vitro produced embryos (Gosden, 2002; Krisher, 2004; Sirard et al., 2006). The vast majority of in vitro matured oocytes are obtained from early antral follicles and thus are meiotically competent; however, many do not attain an optimal oocyte diameter before insemination and also display culture mediainduced anomalies on embryonic development, blastocyst cell number, and apoptosis (Fair et al., 2004; Pennetier et al., 2004; Alizadeh et al., 2005). 


\section{OOCYTE CYTOPLASMIC MATURATION}

Oocyte cytoplasmic maturation includes those events that instill upon the oocyte a capacity to complete nuclear maturation, fertilization, and early embryogenesis and thus provide a foundation for implantation, initiation of pregnancy, and normal fetal development (Brevini-Gandolfi and Gandolfi, 2001; Sirard et al., 2006). Although we can define oocyte cytoplasmic maturation, we are only now beginning to understand the molecular steps that underlie this process (Gosden, 2002).

In general terms, oocyte cytoplasmic maturation involves the accumulation of mRNA, proteins, substrates, and nutrients that are required to achieve oocyte developmental competence that fosters embryonic developmental competence (Brevini-Gandolfi and Gandolfi, 2001; Krisher, 2004; Sirard et al., 2006). Collectively, we are beginning to specify oocyte cytoplasmic maturation, and eventually a coherent understanding of this critical event in oocyte biology will emerge.

\section{OOCYTE MATURATION: TRANSCRIPTIONAL EVENTS}

Similar to invertebrate oocytes, during oogenesis mammalian oocytes accumulate and store large amounts of maternal mRNA that are critical for embryonic development (Brevini-Gandolfi et al., 1999; Brevini-Gandolfi and Gandolfi, 2001; Piccioni et al., 2005). Transcription and storage of maternal mRNA occurs during follicular growth but ceases as the germinal vesicle undergoes breakdown during meiosis (Brevini-Gandolfi et al., 1999; Brevini-Gandolfi and Gandolfi, 2001; Piccioni et al., 2005). The embryo is dependent on stored maternal mRNA until at least the maternal-zygotic transition, when transcription of embryonic genes begins in earnest, with a major burst of transcription occurring at the 2-cell stage in mice (Brevini-Gandolfi and Gandolfi, 2001; Gosden, 2002). There is a largescale degradation of maternal mRNA stores and an increase in mRNA deadenylation during oocyte maturation, and further losses of maternal mRNA occur during early cleavage stages (Brevini-Gandolfi et al., 1999; Brevini-Gandolfi and Gandolfi, 2001; Piccioni et al., 2005). In general, polyadenylation is associated with translational activation, whereas deadenylation is associated with translational silencing (Piccioni et al., 2005).

Only a very few studies have begun to examine the influences of varying culture environments on mRNA deadenylation and mRNA turnover/stability (BreviniGandolfi et al., 1999; Brevini-Gandolfi and Gandolfi, 2001). In addition, there is great need to understand the role of RNA binding proteins in regulating mRNA stability because these proteins represent reasonable targets for culture-induced influences on embryonic mRNA half-life. This will be an important area of future research for this field.

\section{CULTURE INFLUENCES ON EMBRYONIC DEVELOPMENT}

Steady progress in developing improved culture conditions for preimplantation embryos from a variety of mammalian species has occurred over the past $40 \mathrm{yr}$ (Gosden, 2002; Krisher, 2004; Sirard et al., 2006). This rate of progress has intensified in particular in the last 10 to $15 \mathrm{yr}$, resulting in the characterization of effective defined or semidefined media for early embryos in species ranging from the mouse (potassium simplex optimized medium), to sheep and cow (citrate supplemented-synthetic oviduct fluid medium), and most recently the human (G1/G2 formulations). Despite all of the effort that has been applied to their successful development, much of the credit for the success of these media in supporting the first week of development in vitro must still be directed at the quality of the originating oocyte and the early embryo itself.

Preimplantation mammalian embryos display an impressive capacity to adapt to the pressures that suboptimal culture environments place upon them. The embryo can compensate, at least partially, for missing components or offset the presence of deleterious components by adjusting its developmental program (Fair et al., 2004; Pennetier et al., 2004; Alizadeh et al., 2005). This embryonic developmental plasticity operates within a defined range of tolerances that, in fact, may be established in the oocyte during oogenesis and is likely reflected by oocyte developmental competence (Gilchrist et al., 2004).

It has only been within the past decade that, for most mammalian species under investigation, oocyte and embryo culture media have supplied appropriate conditions that fall within these embryonic tolerance zones. Current media, even with their impressive capacities to support preimplantation development in vitro, must still be considered to be suboptimal for all species. We have yet to define all of the vital components of the maternal environment and mechanisms of oogenesis that are required to support early embryonic development, and we are even further from understanding their interactions with one another in supporting early development.

Because of all of these concerns, great effort has focused on understanding how oocyte-cumulus cell interactions are regulated (Gilchrist et al., 2004) and how early embryos adjust their developmental program to compensate for exposure to suboptimal culture environments (Gosden, 2002; Krisher, 2004; Sirard et al., 2006). Approaches in this research area have included studies directly comparing the levels of marker gene expression and also variations in embryonic development between in vitro and in vivo derived preimplantation embryos (Fair et al., 2004; Pennetier et al., 2004; Alizadeh et al., 2005). The primary interpretation of the majority of these studies is that gene transcription is influenced by exposure to varying culture environments. 


\section{SUMMARY}

Research is required to understand the causative events underlying oocyte maturation and early embryonic development (a) so losses can be prevented, (b) to ensure that reproductive technologies are applied in the safest and most efficient ways possible, (c) to define and optimize culture environments, (d) to increase developmental frequencies of embryos from animal species for commercial applications, and (e) to define the mechanisms regulating early development. Many of these goals will be attained as we continue to research and understand the mechanisms controlling the attainment of full oocyte developmental competence, which must rely upon the completion of oocyte cytoplasmic maturation and proper processing, storage, and activation of maternal mRNA.

\section{LITERATURE CITED}

Alizadeh, Z., S.-I. Kageyama, and F. Aoki. 2005. Degradation of maternal mRNA in mouse embryos: Selective degradation of specific mRNAs after fertilization. Mol. Reprod. Dev. 72:281-290.

Bilodeau-Goeseels, S., and P. Panich. 2002. Effects of oocyte quality on development and transcriptional activity in early bovine embryos. Anim. Reprod. Sci. 71:143-155.
Brevini-Gandolfi, T. A. L., L. A. Favetta, L. Mauri, A. M. Luciano, F. Cillo, and F. Gandolfi. 1999. Changes in poly(A) tail length of maternal transcripts during in vitro maturation of bovine oocytes and their relation with developmental competence. Mol. Reprod. Dev. 52:427-433.

Brevini-Gandolfi, T. A. L., and F. Gandolfi. 2001. The maternal legacy to the embryo:cytoplasmic components and their effects on early development. Theriogenology 55:1255-1276.

Fair, T., M. Murphy, D. Rizos, C. Moss, F. Martin, M. P. Boland, and P. Lonergan. 2004. Analysis of differential maternal mRNA expression in developmentally competent and incompetent bovine two-cell embryos. Mol. Reprod. Dev. 67:136-144.

Gilchrist, R. B., L. J. Ritter, and D. T. Armstrong. 2004. Oocytesomatic cell interactions during follicle development in mammals. Anim. Reprod. Sci. 82-83:431-446.

Gosden, R. G. 2002. Oogenesis as a foundation for embryogenesis. Mol. Cell. Endocrinol. 186:149-153.

Krisher, R. L. 2004. The effect of oocyte quality on development. J. Anim. Sci. 82(Suppl. E):E14-E23.

Pennetier, S., S. Uzbekova, C. Perreau, P. Papillier, P. Mermillod, and R. Dalbies-Tran. 2004. Spatio-temporal expression of the germ cell marker genes Mater, ZAR1, GDF9, BMP15 and VASA in adult bovine tissues, oocytes and preimplantation embryos. Biol. Reprod. 71:1359-1366.

Piccioni, F., V. Zappavigna, and A. C. Verrotti. 2005. Translational regulation during oogenesis and early development: The cappoly(A) tail relationship. C.R. Biologies 328:863-881.

Sirard, M.-A., F. Richard, P. Blondin, and C. Robert. 2006. Contribution of the oocyte to embryo quality. Theriogenology 65:126-136. 